\title{
MOLUSCOS POLIPLACÓFOROS DEL LITORAL ATLÁNTICO DEL SUR DE LA PENÍNSULA IBÉRICA
}

\author{
M. P. Carmona Zalvide (*) y F. J. García (**)
}

\begin{abstract}
RESUMEN
Se aporta el catálogo de los Moluscos Poliplacóforos de las costas atlánticas del sur de la Península Ibérica, desde Sagres (Portugal) hasta Gibraltar. Se cita un total de 20 taxones (Lepidopleurus cajetanus, Leptochiton cancellatus, Leptochiton algesirensis, Leptochiton scabridus, Callochiton septemvalvis, Callochiton euplaeae, Lepidochitona cinerea, Lepidochitona corrugata, Lepidochitona canariensis, Lepidochitona monterosatoi, Lepidochitona kaasi, Lepidochitona severianoi, Chaetopleura angulata, Ischnochiton rissoi, Chiton olivaceus, Chiton corallinus, Chiton phaesolinus, Acanthochi-tona fascicularis y Acanthochitona crinita) todos ellos pertenecientes al dominio litoral. La captura de Lepidochitona canariensis y L. simrothi en aguas atlánticas ibéricas constituye la primera cita para el suratlántico ibérico. A su vez se amplía la distribución a esta zona de Callochiton septemvalvis y de Lepidochitona monterosatoi.

Palabras claves: Moluscos poliplacóforos, catálogo faunístico, distribución litoral, SO Ibérico.
\end{abstract}

\begin{abstract}
Catalogue of the Mollusca Polyplacophora from the Atlantic coast of Southern Iberian Peninsula

In this paper, an updated check-list of the polyplacophoran species from Sagres (Portugal) to Strait of Gibraltar is present. Twenty taxa are recorded in this area: Lepidopleurus cajetanus, Leptochiton cancellatus, Leptochiton algesirensis, Leptochiton scabridus, Callochiton septemvalvis, Callochiton euplaeae, Lepidochitona cinerea, Lepidochitona corrugata, Lepidochitona canariensis, Lepidochitona monterosatoi, Lepidochitona kaasi, Lepidochitona severianoi, Chaetopleura angulata, Ischnochiton rissoi, Chiton olivaceus, Chiton corallinus, Chiton phaesolinus, Acanthochitona fascicularis and Acanthochitona crinita. From these species, Lepidochitona canariensis, L simrothi, Callochiton septemvalvis and Lepidochitona monterosatoi amplify their geographical distribution to Southern Atlantic coast of southern Iberian Peninsula.

Key words: Molluscan Polyplacophora, check-list, Atlantic coast, SW Iberian Peninsula.
\end{abstract}

\section{Introducción}

No son muy abundantes los estudios taxonómicos sobre Poliplacóforos en las costas de la Península Ibérica. Generalmente, se incluyen dentro de listados de Moluscos de una zona determina-

da, posiblemente debido a la confusa taxonomía que presentaba este grupo hasta hace poco tiempo y por el escaso número de especies que se encuentran en la Península Ibérica en comparación con otros grupos faunísticos.

* Departamento Bioloxía Animal, Facultade de Bioloxía, Universidade de Santiago. 15706 Santiago de Compostela.

** Laboratorio de Biología Marina, Departamento de Fisiología y Biología Animal, Facultad de Biología, Universidad de Sevilla, Avda. Reina Mercedes s/n, Apdo.1095, 41080 Sevilla. 
Los primeros datos que se tienen de trabajos efectuados en las costas ibéricas se remontan a 1849, fecha en la que MacAndrew (1849) estudia los Moluscos de Vigo. Posteriormente, dicho autor amplia su estudio a las costas portuguesas, Gibraltar y costas mediterráneas (MacAndrew, 1850).

Capellini (1859) estudió material procedente de Algeciras (Cádiz) y describió como nueva especie para la ciencia a Leptochiton (L.) algesirensis. Posteriormente Monterosato (1888) también recibió material de Gibraltar. Hidalgo (1867) fue el primer español que estudió los moluscos poliplacóforos de la Península Ibérica. Posteriormente este autor realizó un trabajo complementario en el que amplió el número de especies ibéricas (Hidalgo, 1917).

Las costas suratlánticas ibéricas han sido poco estudiadas. Destacan los trabajos de Reis y Montero (1984), que estudian los poliplacóforos del Algarve, Aartsen et al. (1984) que estudian la fauna malacológica de Algeciras y Estacio et al. (1992) la del Río Piedras (Huelva).

En el presente trabajo se aporta un listado de los Moluscos Poliplacóforos de las costas atlánticas del sur de la Península Ibérica desde Sagres (Portugal) hasta Gibraltar. Se citan un total de 20 especies y se incluye una breve descripción de ellas.

\section{Material y métodos}

Los ejemplares estudiados han sido recolectados en el litoral Suratlántico de la Península Ibérica, distribuidas desde Praia Mareta (Sagres, Portugal) hasta Punta de Europa, en el Estrecho de Gibraltar (Fig. 1). La relación de las localidades de muestreo se especifica en el Apéndice I. Los ejemplares se capturaron tanto en la zona intermareal como en la infralitoral, en ésta con equipo de inmersión autónoma. Posteriormente fueron relajados con cristales de mentol y fijados, entre dos portaobjetos con etanol absoluto, seguidamente fueron conservados en alcohol 70\% para su posterior determinación.

\section{Resultados y discusión}

Clase POLYPLACOPHORA Gray, 1821

Orden NEOLORICATA Bergenhayn, 1955

Suborden LEPIDOPLEURINA Thiele, 1910

Familia LEPTOCHITONIDAE Dall, 1889

Lepidopleurus cajetanus Poli, 1791

Material estudiado: E 34: 1ej., VI/93 (9 m); 6 ej., IX/93 (10 m); 1 ej., XI/93 (8 m). E 40: 1 ej., VII/91 (20 m). E 43: 2 ej.,

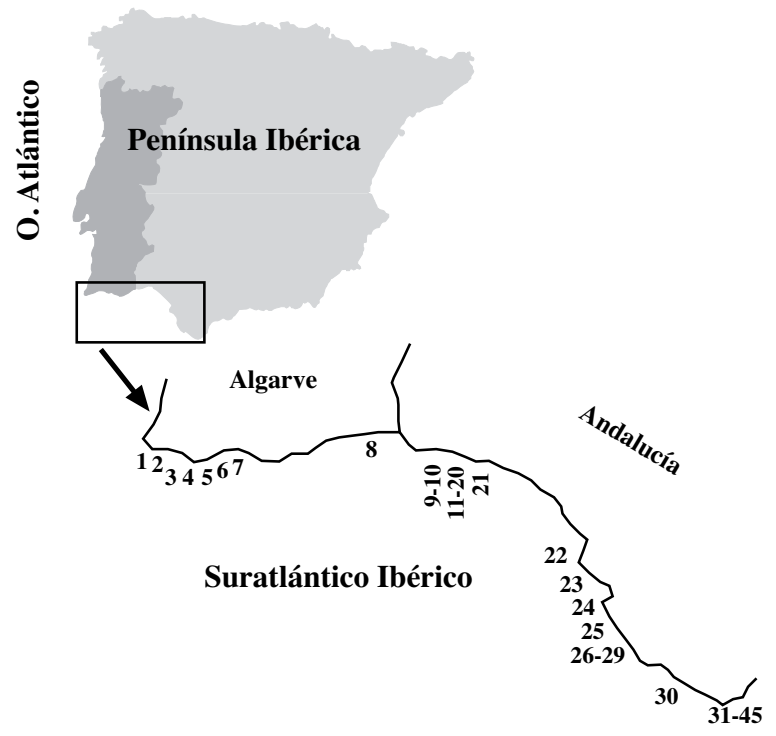

Fig. 1.- Estaciones de muestreo numeradas como en el Apéndice.

Fig. 1.- Collecting stations.

VII/92 (33 m). E 45: 1 ej., IV/93 (12 m); 1 ej., VII/93 (11 m); 3 ej., IX/93 (12 m); 1 ej., I/94 (10 m).

Citas bibliográficas: Algeciras (Jeffreys, 1882; Hidalgo, 1917; Aartsen et al., 1984); Portugal (Capellini, 1859; Locard, 1886; Bucquoy et al., 1882); Olhao (Nobre, 1938); costas atlánticas de la Península Ibérica (Sabelli, 1974); Portugal (Boggi et al., 1980); Portimao (Reis y Monteiro, 1984); Golfo de Cádiz (Kaas y Van Belle, 1985a).

El tamaño de los ejemplares estudiados oscila entre $2,5 \times 1,8 \mathrm{~mm}$ y 22,1 x $9,3 \mathrm{~mm}$. El cuerpo es ovalado, con las placas de la concha muy consistente, no carenados. La ornamentación de éstas se encuentra constituida por gruesos y toscos tubérculos que forman cadenas, dispuestas en sentido longitudinal en el área central y radial en las áreas pleurales y placa cefálica caudal. En estas zonas se distinguen gruesos cordones concéntricos desde el ápice hasta el margen terminal de las placas, que en ejemplares adultos se puede apreciar hasta 8 cordones. El estrecho perinoto se encuentra constituido por pequeños corpúsculos no imbricados.

Esta especie es poco frecuente en las costas atlánticas de la Península Ibérica (Borja, 1983; Burnay, 1986), pudiéndose considerar como rara, e incluso Burnay (1986) aclara que puede ser debido a que requiera un biotopo específico. 


\section{Leptochiton (Leptochiton) cancellatus (Sowerby,} 1840)

Material estudiado: E 06: 1 ej., ViII/93 (22 m). E 30: 1 ej., I/93 (15 m). E 32: 1 ej., VII/91 (9 m); 1 ej., IX/91 (18 m). E 33: 1 ej., IV/92 (9 m); 16 ej., X/92 (12 m). E 34: 1 ej., VI/93; 2 ej., X/93 (10 m); 2 ej., XI/93 (9 m). E 35: 1 ej., I/93 (9 m); 1 ej., VI/93 (5 m); 1 ej., XII/93 (5 m); 1 ej., II/94 (5 m). E 36: 9 ej., VII/93 (2 m). E 37: 2 ej., VII/93 (10 m). E 38: 2 ej., VII/91 (16 m). E 41: 6 ej., VIII/92 (18-20 m). E 43: 3 ej., VII/91 (3 m); 1 ej., VIII/91 (8 m); 83 ej., VII/94 (13-17 m). E 44: 1 ej., $\mathrm{VII} / 91(6 \mathrm{~m})$

Citas BIBliográficas: Costas atlánticas de la Península Ibérica (Malatesta, 1962); Algeciras (Aartsen et al., 1984); Portugal (Reis y Monteiro, 1984).

El tamaño ha oscilado entre $2,1 \times 1,3 \mathrm{~mm}$ y 7,4 x 3,2 $\mathrm{mm}$. El aspecto es ovalado, no carenado y de valvas redondeadas, en las que no se aprecia el ápice. La ornamentación la constituyen gránulos achatados, dispuestos en quincunce, que tienden a unirse para formar cadenas. Se aprecia claramente líneas de crecimiento concéntricas en las valvas. El perinoto es estrecho y erguido por corpúsculos redondeados no imbricados. El fleco marginal es poco patente.

\section{Leptochiton (Leptochiton) algesirensis (Capellini, 1859)}

Material estudiado: E 02: 1 ej., VIII/93 (7 m). E 03: 3 ej., VIII/93 (17 m). E 30: 3 ej., VI/91 (intermareal); 4 ej., IV/92 (4-10 m); 1 ej., VII/92 (2 m); 34 ej., I/93 (9 m). E 31: 2 ej., VIII/91 (3-12 m). E 32: 4 ej., V/90 (intermareal). E 34: 1 ej., IX/92 (12 m); 1 ej., IV/93 (5 m); 1 ej., VII/93 (10 m); 2 ej., VIII/93 (10 m); 1 ej., XI/93 (5 m); 1 ej., I/94 (10 m); 2 ej., II/94 (10 m); E 35: 1 ej., IV/93 (5 m); 1 ej., V/93 (5 m); 3 ej., VI/93 (5 m); 1 ej., VII/93 (5 m); 4 ej., X/93 (5 m); 1 ej., XI/93 (5 m); 1 ej., I/94 (12 m); 4 ej., II/94 (5 m). E 36: 3 ej., VII/93 (9 m). E 37: 2 ej., VII/91 (3 m). E 38: 1 ej., VII/90 (16 m). E 43: 1 ej., VII/91 (33 m).

Citas BIBliográficAs: Algeciras (Capellini, 1859; Monterosato, 1879; Aartsen et al., 1984); Portugal (Bucquoy et al., 1882; Locard, 1886; Bogi et al., 1980; Kaas y Van Belle, 1980; Piani, 1980); Algeciras y Gibraltar (Monterosato, 1888); Lagos, Algeciras y Gibraltar (Hidalgo, 1917); Lagos (Nobre, 1932); Lagos y Olhao (Nobre; 1938); costas atlánticas de la Península Ibérica (Malatesta, 1962; Sabelli, 1974); Algarve y Gibraltar (Kaas y Van Belle, 1985a).

El tamaño de los ejemplares ha variado entre 2,2 x $1,8 \mathrm{~mm}$ y 21,3 x $12,8 \mathrm{~mm}$. Cuerpo ovalado, con la zona caudal más ancha que la oral; no carenado y ápices no marcados, con concha poco consistente. El tegmento se encuentra ornamentado por finos gránulos que se unen y forman cadenas. Éstas se encuentran interrumpidas por las líneas de crecimiento, que se disponen en semicírculos concéntricos en las valvas terminales y áreas laterales. El perinoto está constituido por escamas no imbrica- das de aspecto redondeado, ornamentadas con costillas.

Leptochiton algesirensis ha sido encontrado en la zona intermareal bajo piedras sueltas de roquedos, localizadas siempre en charcas de mareas con Corallina elongata y Asparagopsis cuneata, no encontrándose nunca los ejemplares en emersión. En la zona infralitoral, se han recolectado hasta 17 $\mathrm{m}$ de profundidad y aparecían asociados a piedras sueltas, tanto de roquedo como de arenales, con gran cobertura de algas calcáreas.

Leptochiton (Leptochiton) scabridus (Jeffreys, 1880) Material estudiado: E 34: 2 ej., II/94 (8 m).

Citas BibliográficAs: Algeciras (Aartsen et al., 1984); Portugal (Kaas y Van Belle, 1987).

El cuerpo es ovalado, no carenado y con ápices no marcados; el dorso es de forma redondeada. La ornamentación es patente, constituida por gruesos tubérculos toscos, de apariencia redondeada, que tienden a formar cadenas lineales. El estrecho perinoto está constituido por escamas imbricadas de aspecto redondeado. El fleco marginal es poco patente.

Es una especie rara en el litoral suratlántico, únicamente se han capturado dos ejemplares, adosados a la cara inferior de piedras aisladas, que se encontraban en fondos arenosos.

Suborden ISCHNOCHITONINA Bergenhayn, 1930 Familia ISCHNOCHITONIDAE Dall, 1889

\section{Ischnochiton (Ischnochiton) rissoi (Payraudeau, 1826)}

Material estudiado: E 29: 6 ej., VII/92 (intermareal). E 30: 1 ej., VII/90 (7 m); 6 ej., III/91 (10 m); 1 ej., IV/92 (3 m); 6 ej., I/93(9 m). E 31: 4 ej., VIII/91(8 m); 9 ej., IX/92 (12 m); 1 ej., XI/93 (9 m). E 33: 10 ej., IV/92 (5-9 m). E 34: 1 ej., VII/91 (4$5 \mathrm{~m}) ; 14$ ej., XI/91 (5 m); 1 ej., IX/92 (12 m); 6 ej., IX/92 (12 m). E 35: 3 ej., IX/92 (3 m); 3 ej., IX/92 (5 m); 81 ej., I/93 (5 m); 1 ej., IX/93 (3 m). E 36: 1 ej., VII/93 (5 m). E 37: 1 ej., VII/91 (5 m); 1 ej., VII/91 (3 m); 2 ej., VIII/91 (6 m); 1 ej., VII/93). E 38: 1 ej., VII/90 (14 m); 7 ej., VII/91 (9 m); 1 ej., VII/91 (16 m). E 40: 1 ej., VIII/92 (5 m). E 44: 2 ej., VII/91 (5 m). E 45: 1 ej., IV/92 (25 m); 1 ej., VII/93 (15 m).

Citas BIBLIOGRÁFICAS: Gibraltar (Hidalgo, 1917); Algeciras (Van Aartsen et al., 1984).

Se han recolectado ejemplares de tamaños comprendidos entre 3 × 1,5 y $18 \times 9 \mathrm{~mm}$. El aspecto del cuerpo es oblongo, ligeramente carenado, regularmente arqueado, con conchas consistentes, que exhiben una gran variabilidad en la ornamentación del tegmento. El perinoto es relativamente ancho, 
constituido por escamas imbricadas con apariencia de piel de serpiente y con un fleco marginal patente.

Se han encontrado en piedras sueltas de zonas rocosas, aunque también en algunas ocasiones en arenosas, todas las piedras se caracterizaban por estar cubiertas de algas calcáreas.

\section{Familia CALLOCHITONIDAE Thiele, 1910}

\section{Callochiton septemvalvis (Montagu, 1803)}

MATERial estudiado: E 04: 1 ej., VIII/88 (intermareal). E 34: 2 ej., IX/92 (12 m).

El tamaño de los ejemplares estudiado ha variado entre 3,7 × 2 y 8 × 4,5 mm. El tegmento es granulado, en el que destaca los punteados negros de la copa de los pigmentos de las estetas, dispuestos de forma radial en las áreas laterales de las placas intermedias y en la zona postmucral de la placa caudal. Las apófisis se encuentran conectadas a través del seno jugal. El perinoto es bastante ancho, provisto de un fleco de espículas marginales, pudiendo presentar espículas supramarginales curvadas aisladas o en grupo.

La captura de Callochiton septemvalvis en aguas suratlánticas ibéricas constituye la primera cita para esta localidad.

\section{Callochiton euplaeae (O.G. Costa, 1829)}

Material estudiado: E 24: 1 ej., Vit/92 (12 m). E 29: 2 ej., VII/92 (5 m). E 31: 4 ej., IX/92 (12 m). E 32: 2 ej., VIII/91 (6 m). E 33: 1 ej., VIII/91 (12 m). E34: 2 ej., VII/91 (9 m); 3 ej., IX/92 (12 m); 1 ej., V/93 (12 m); 3 ej., VI/92 (9 m); 1 ej., VII/93 (10 m); 1 ej., $(9 \mathrm{~m}) ; 5$ ej., X/93 (8 m). E 35: 1 ej., I/93 (5 m). E 37: 2 ej., VIII/91 (6 m). E 38: 1 ej., VII/90 (9 m); 1 ej., VIII/91 (3 m). E 43: 2 ej., IV/93 (10 m); 4 ej., VII/93 (9 m); 1 ej., IX/93 (9 m). E 45: 1 ej., IV/93 (10 m); 1 ej., VII/93 (12 m); 1 ej., VIII/93 (8 m); 1 ej., XI/93 (12 m).

CitAS BIBLIOGRÁfiCAS: Algeciras (Aartsen et al., 1984).

El tamaño de los ejemplares recolectados ha variado entre 2 x 1,5 y 18 x $10 \mathrm{~mm}$. El cuerpo es de forma ovalada, carenado, con ápices bien marcados. El tegmento de las valvas terminales y áreas laterales presenta puntos negros, que corresponden a las copas de los pigmentos de las estetas. Las placas ofrecen una ornamentación de cinco costillas, dispuestas en las áreas pleurales de las placas intermedias y en la zona anteromucral de la placa caudal. El perinoto es bastante ancho, constituido por espículas alargadas imbricadas, sobresaliendo dorsalmente espinas curvadas.

Callochiton euplaeae suele ser una especie solitaria, normalmente se encuentran uno o dos indivi- duos. Se ha capturado en la cara inferior de piedras sueltas, generalmente en roquedos localizados en facies de Halopteris.

\section{Familia CHAETOPLEURIDAE Moore, 1960}

\section{Chaetopleura angulata (Spengler, 1797)}

Material estudiado: E 08: 3 ej., IV/80. E 09: 2 ej., VII/91 (intermareal). E 10: 2 ej., V/92 (14 m). E 11: 1 ej., VII/87 (5 m). E 12: 1 ej., IX/88 (5 m). E 13: 3 ej., XI/87 (3-4 m); 2 ej., I/88 (3-4 m); 3 ej., III/88 (3-4 m); 1 ej., V/88 (3-4 m); 3 ej., IX/88 (5 m); 1 ej., XI/88 (3-4 m). E 14: 1 ej., IX/87 (3-4 m). E 15: 4 ej., V/87 (5 m). E 16: 1 ej., XI/88 (5 m). E 17: 1 ej., VIII/88 (intermareal). E 18: 1 ej., VII/87 (intermareal); 116 ej., II- XII/93 (intermareal); 13 ej., I/94 (intermareal). E 19: 10 ej., III/91 (intermareal). E 20: 2 ej., VII/92 (intermareal). E 21: 3 ej., X/92 (35 m). E 22: 12 ej., III/92 (intermareal); 45 ej., $48 \mathrm{x}$ 23 mm.IV/92 (intermareal). E 26: 1 ej., IX/92 (28 m).

CitAS BIBlIOGRÁFICAS: Portugal (Wood, 1815; Pilsbry, 1892; Reis y Monteiro, 1984; Kaas y Van Belle, 1987); costas de España y Portugal (Reeve, 1847); Cádiz y Gibraltar (Hidalgo, 1917); Faro de Olhao (Nobre, 1938); costas atlánticas de la Península Ibérica (Kaas, 1954); Río Piedras (Estacio et al., 1993).

El tamaño de los ejemplares ha variado entre 8 x 5 y 60 x $28 \mathrm{~mm}$. Ofrecen una forma oval, fuertemente carenado, con ornamentación sobre las placas de cadenas longitudinales en las áreas intermedias y pústulas en las laterales y valvas extremas. Perinoto ancho, de aspecto carnoso, sin fleco patente de espículas marginales.

En las costas suratlánticas, ser han recolectado ejemplares desde la zona intermareal, en playas areno fangosas, donde constituyen poblaciones abundantes sin convivir con ninguna otra especie de poliplacóforos, hasta los $25 \mathrm{~m}$ de profundidad, también en fondos fangosos, adosados a valvas de ostras principalmente, y nunca se ha encontrado con otra especie de poliplacóforo acompañante.

\section{Familia LEPIDOCHITONIDAE Iredale, 1914 \\ Lepidochitona cinerea (Linneo, 1767)}

Material estudiado: E 02: 6 ej., VIII/93 (7 m). E 03: 1 ej., VIII/93 (17 m). E 05: 1 ej., IV/88 (intermareal); 4 ej., VIII/88 (intermareal). E 07: 10 ej., VIII/93 (intermareal). E 16: 1 ej., V/88 (intermareal). E 18: 1 ej., VII/87 (intermareal); 4 ej., V/92 (intermareal); 6 ej., III/93 (intermareal). E 20: 3 ej., IV/91 (intermareal). E 23: 1 ej., XI/90 (intermareal). 1 ej., VI/92 (intermareal). E 25: 1 ej., IX/95 (intermareal). E 27: 1 ej., IV/94 (intermareal). E 28: 2 ej., VII/92 (5-6 m). E 29: 26 ej., VII/92 (intermareal). E 30: 1 ej., II/91 (3 m); 2 ej., I/93 (intermareal). E 33: 4 ej., V/91 (intermareal). E 42: 5 ej., III/91 (intermareal); 9 ej., VIII/91 (intermareal).

Citas BIBliográficas: Sur de Portugal (MacAndrew, 1849; 1850); Portugal (Forbes y Hanley, 1853; Bucquoy et al., 1882; 
Jeffreys, 1882; Piani, 1980); Algeciras (Capellini, 1859; Aartsen et al., 1984); Gibraltar y Algeciras (Monterosato, 1888); Algarve, Cádiz y Algeciras (Hidalgo, 1917); Ría de Faro y Olhao (Nobre, 1932, 1938-40); costas atlánticas de la Península Ibérica (Malatesta, 1962; Sabelli, 1974); Algarve (Kaas y Van Belle, 1981; Reis y Monteiro, 1984); Portugal y Andalucía (Kaas y Van Belle, 1985ab); Río Piedras (Estacio et al., 1993).

El tamaño de los ejemplares recolectados ha variado entre $1,6 \times 1$ y 15 x $9 \mathrm{~mm}$. El aspecto general del animal es ovalado y algo achatado, carenado, ápice bien marcado, con conchas consistentes nacaradas. El tegmento se encuentra ornamentado por gránulos alargados y redondeados, dispuestos en quincunce. Las líneas de crecimiento están bien marcadas en las áreas laterales de las placas intermedias y en las placas cefálica y caudal. El perinoto está constituido, dorsalmente, por pequeños corpúsculos imbricados.

Lepidochiton cinerea es una especie muy común y abundante en el litoral suratlántico de la Península, su principal hábitat es la zona mediolitoral, adosadas a piedras bien de roquedos o bien de playas arenosas, aunque también se ha capturado hasta $17 \mathrm{~m}$ de profundidad. Generalmente se encuentra asociada con otras especies de poliplacóforos, siendo más corriente con Acanthochitona crinita y Chiton olivaceus.

\section{Lepidochitona corrugata (Reeve, 1848)}

MATERIAL ESTUdiado: E 23: 10 ej., VI/92 (intermareal); 8 ej., III/94 (intermareal). E 30: 2 ej., V/91 (intermareal); 1 ej., VI/91 (intermareal). E 32: 8 ej., V/91 (intermareal). E 33: 1 ej., V/91 (intermareal). E 37: 4 ej., VIII/91 (6 m); E 42: 1 ej., VIII/91 (intermareal). E 43: 2 ej., VI/93 (10 m); 1 ej., VII/93 (10 m).

CitAS BIBLIOGRÁFICAS: Portugal (Capellini, 1859; Bucquoy et al., 1882; Locard, 1886); Lagos, Ría de Faro, Olhao y Cádiz (Pilsbry, 1892); Cádiz (Maluquer, 1915); océano Atlántico ibérico (Thiele, 1929); Lagos, Ría de Faro, Olhao (Nobre, 193840); costas suratlánticas de la Península Ibérica (Malatesta, 1962); costas meridionales atlánticas españolas (Sabelli, 1974); Algarve y Golfo de Cádiz (Kaas y Van Belle, 1981, 1985b); Algarve (Reis y Monteiro, 1984); Algeciras (Aartsen et al., 1984).

El tamaño de los ejemplares recolectados ha variado entre $3,1 \times 2,5$ y 14 × $9 \mathrm{~mm}$. El aspecto general del animal es ovalado, no carenado, regularmente arqueado, con conchas consistentes. El tegmento se encuentra ornamentado por gruesos y toscos gránulos, que en individuos adultos, suelen estar desgastados en las zonas más viejas de las valvas.

Los ejemplares de L. corrugata se encuentran principalmente en la zona intermareal adosados a la parte inferior de piedras, bien en zonas areno-fangosas y con algas del género Enteromorpha o bien, en piedras de cubetas de roquedos. En la infralitoral, es menos frecuente, aunque también aparece bajo piedras aisladas de estaciones arenosas en facies de Halopteris.

Lepidochitona simrothi (Thiele, 1902)

Material estudiado: E 05: 6 ej., IV/88 (intermareal).

Ovalado, subcarenado, con los lados redondeados y ápices algo marcados. El tegmento se encuentra ornamentado por tubérculos prominentes de apariencia tosca. El perinoto es granuloso, a veces provisto de espinas dorsales dispuestas en solitario y con un fleco marginal patente. El tamaño de los ejemplares estudiado ha variado entre $3,5 \times 1$ y 9,8 $\mathrm{x} 7,7 \mathrm{~mm}$.

A Lepidochitona simrothi (Thiele, 1902) se la denominó como Nutallina (Middendorffia) simrothi. Desde su descripción no se había vuelto a recolectar hasta la expedición de Jean Charcot Biaçores (1971) y por M. Lavaleye y Frias Martins, en las Azores, ejemplares que publican Kaas y Van Belle (1981). El hallazgo de esta especie constituye la primera cita para la Península Ibérica.

Lepidochitona canariensis (Thiele, 1909)

Material estudiado: E 01: 6 ej., VII/94 (8 m). E 30: 4 ej., VI/91 (intermareal). E33: 2 ej., V/91 (intermareal); 1 ej., IX/91 (12 m). E 37: 1 ej., VIII/91 (6 m). E 43: 3 ej., III/93 (10 m); 1 ej., V/93 (10 m); 1 ej., VI/93 (10 m); 1 ej., IX/93 (10 m).

El tamaño de los ejemplares capturados oscila entre 3,5 × $2 \mathrm{~mm}$ y 7,2 × $4 \mathrm{~mm}$. El cuerpo es ovalado, subcarenado, con las placas rectangulares, provistas de un ápice prominente y con los lados ligeramente convexos, lo cual ofrece al animal un aspecto curvado dorsalmente. La ornamentación de las placas es de aspecto algo tosco. El perinoto es relativamente estrecho, constituido por pequeños corpúsculos; a veces también presentan espículas curvadas. Las espículas marginales constituyen un fleco bastante sobresaliente.

Esta especie ha sido encontrada bajo piedras sueltas, recubiertas por algas calcáreas, tanto en la zona intermareal de playas arenosas con rocas, como en la infralitoral hasta $10 \mathrm{~m}$ de profundidad, bien en roquedos o bien en fondos biodetríticos.

Lepidochitona canariensis no se había recolectado en aguas ibéricas por lo que se considera la primera cita para la Península Ibérica.

Lepidochitona monterosatoi Kaas y Van Belle, 1981

Material estudiado: E 01: 1 ej., VII/94 (5 m). E 30: 1 ej., VI/91 (intermareal). E 33: 2 ej., V/91 (intermareal). E 37: 3 ej., VIII/90 (6 m). 39: 1 ej., VII/90 (3 m). E 43: 1 ej., IV/93 (11 m). 
El tamaño de los ejemplares capturados ha variado entre $3,5 \times 2 \mathrm{~mm}$ y 7,5 x 5,5 $\mathrm{mm}$. El cuerpo es ovalado, subcarenado, con las valvas provistas de un ápice marcado, que ofrece un aspecto picudo. El tegmento se caracteriza por tener una granulación algo prominente y de apariencia tosca. El perinoto es granulado, a veces provistos de espinas dorsales dispuestas en solitario o en grupos. Las espículas del fleco marginal se encuentran surcadas longitudinalmente.

Desde su descripción L. monterosatoi ha sido capturada únicamente en el Mar Mediterráneo y en las costas gallegas (Carmona y Urgorri, 1999) los ejemplares capturados en el sur atlántico Ibérico, permiten ampliar su distribución geográfica hasta aguas suratlánticas ibéricas, constituyendo la primera cita de dicha especie para esta zona.

\section{Lepidochitona kaasi Carmona y García, 2000}

Material estudiado: E 30: 22 ej., VI/91 (intermareal); 3 ej., VII/95 (intermareal).

El tamaño es pequeño de hasta $3,5 \times 2 \mathrm{~mm}$. El aspecto que presentan es ovalado, con las placas redondeadas no carenadas y con el ápice muy marcado. La coloración es parda, a veces con manchas blancas. La ornamentación está constituida por gránulos toscos redondeados. El perinoto es estrecho, con bandas alternas en dos colores claro y oscuro, en el que sobresale el fleco de espículas marginales. El diente mayor marginal de la rádula presenta la placa uncinada tricúspide con los tres dentículos de tamaños prácticamente iguales, aunque sobresale ligeramente el central.

Solamente se han recolectado ejemplares en pozas de roquedos de la zona intermareal de la Isla de tarifa.

\section{Lepidochitona severianoi Carmona y García, 2000}

MATERIAL ESTUdiado: E 27: 1 ej., IX/93 (intermareal); E 31: 1 ej., IX/92 (12 m); E 32: 1 ej., V/91 (intermareal); E 33: 1 ej., V/91 (intermareal); 1 ej., IX/91 (12 m); E 43: 1 ej., V/93 (10 m); 1 ej., VI/93 (10 m); 1 ej., X/93 (10 m).

El tamaño máximo de los ejemplares es de 9 $\mathrm{mm}$ de longitud y $5,2 \mathrm{~mm}$ de anchura. La coloración es rosada con matices anaranjados muy pálidos y manchas pardas en la zona central de las valvas. Forma ovalada, subcarenada, con las áreas laterales patentes y elevadas, aunque normalmente se encuentran muy erosionadas. La ornamentación la forman gránulos redondeados de aspecto tosco, dispuestos en quincunce. Puede ofrecer de 3-4 costillas longitudinales en las áreas pleurales. Presenta 8-9 surcos radiales en la valva cefálica y hasta 3 en las laterales. La placa caudal es de menor tamaño. El aspecto del perinoto es granulado, constituido por corpúsculos no imbricados y lisos, entre los cuales sobresalen espículas aisladas o en penachos de dos o tres. El fleco marginal es poco patente. La placa uncinada del diente mayor lateral de la rádula es tricúspide, sobresaliendo la punta central en los dientes de nueva formación, mientras que en los más antiguos se encuentran al mismo nivel.

Se ha recolectado en zonas rocosas con abundancia de algas calcáreas. Su distribución batimétrica se extiende desde la zona intermareal hasta 10 $\mathrm{m}$ de profundidad.

\section{Familia CHITONIDAE Rafinesque, 1815}

\section{Chiton olivaceus (Spengler, 1797)}

Material estudiado: E 03: 2 ej., ViII/93 (17 m). E 05: 6 ej., VIII/88 (intermareal). E 06: 2 ej., VIII/93 (22 m). E 24: 2 ej.,VII/92 (12 m). E 28: 1 ej.,VII/92 (8 m). E 29: 5 ej., VII/92 (intermareal). E 30: 7 ej., III/91 (10 m); 2 ej.,VII/91 (intermareal). E 31: 1 ej., VII/91 (8 m); 1 ej., VIII/91 (6-8 m); 5 ej., IX/92 (3-12 m). E 32: 1 ej., V/91 (intermareal); 3 ej., VIII/91 (intermareal). E 33: 10 ej., IV/91 (6 m); 1 ej., VII/91 (4 m); 1 ej., VII/91 (4-6); 5 ej., VIII/91 (5 m); 8 ej., IV/92 (12 m). E 34: 1 ej.,VII/90 (4 m); 1 ej.,VII/91 (4-6 m); 2 ej.,VII/91 (6 m); 3 ej.,IX/92 (8 m); 12 ej., IX/92 (12 m). E 35: 11 ej., IX/92 (3 m); 10 ej., I/93(5 m). E 37: 2 ej., VII/91 (10 m); 3 ej., VII/91 (12 m); 21 ej., VIII/91 (6 m); 2 ej., VII/92 (10 m). E 38: 3 ej., VII/90 (12 m); 12 ej., VII/91 (15 m); 1 ej., VIII/91 (16 m). E 39: 1 ej., VII/90 (16 m); 3 ej., VII/91 (25 m). E 40: 5 ej., I/93 (3-6 m). E 42: 1 ej., VII/90 (intermareal); 8 ej., III/91 (intermareal). E 43: 1 ej., VII/90 (6 m); 1 ej., III/91 (6 m); 1 ej., VI/91 (8 m); 5 ej.,VII/91 (10 m); 3 ej., VIII/91 (9 m); 1 ej., II/92 (14 m); 53 ej., IX/93 (33 m). E 44: 3 ej., VII/91 (15 m); 11 ej., VIII/91 (12 m); 1 ej., X/91 (17 m). E 45: 2 ej., VII/92 (20-25 m)

Citas Bibliográficas: Estrecho de Gibraltar (MacAndrew, 1850; Carus, 1883; Laghi, 1977); bahía de Algeciras (Hidalgo, 1917; Van Aartsen et al., 1984); Cádiz (Hidalgo, 1917); Portugal (Bucquoy et al., 1882; Locard, 1886; D’Angelo y Gargiullo, 1978; Kaas y Van Belle, 1980); Algarve (Reis y Montero, 1984)

Se han recolectados ejemplares de tamaños comprendidos entre 3 x 2 y $28,5 \times 15 \mathrm{~mm}$. El aspecto general del animal es ovalado, con una fuerte elevación, carenado, las placas son gruesas y consistentes. Las valvas terminales y áreas laterales ofrecen una estriación radial. En las placas intermedias se encuentran bien delimitadas las diferentes áreas. En la zona pleural presentan una serie de costillas longitudinales muy marcadas que disminuyen en longitud hacia la zona jugal. El perinoto es ancho y constituidos por escamas, que ofrecen un aspecto de piel de serpiente. 
Es una especie muy común en el litoral de Cádiz, que disminuye en número para ser ocasional y rara a medida que se penetra en el Atlántico. Se encuentra en fondos muy diversos tanto de roquedos, arenosos como biodetríticos. Suelen estar acompañados de otras especies de poliplacóforos como C. corallinus, I. rissoi y A. fascicularis.

\section{Chiton corallinus (Risso, 1826)}

Material estudiado: E 30: 1 ej., III/91 (6 m); 2 ej., IV/92 (intermareal). E 31: 1 ej., VIII/91 (5 m); 3 ej., IX/92 (3-12 m); 2 ej., XI/93 (9 m). E 33: 3 ej., IV/92 (12 m). E 34: 1 ej., VII/91 (6 m); 1 ej., IX/92 (8 m); 3 ej., IX/92 (6 m); 1 ej., III/93 (4-6 m); 1 ej., VI/93 (8 m); 2 ej., VII/93 (6 m); 1 ej., VIII/93 (6 m); 4 ej., IX/93 (12 m); 5 ej., XII/93 (8 m); 1 ej., I/94 (5 m); 4 ej., II/94 (5 m). E 35: 2 ej., I/93 (12 m); 1 ej., XI/93 (5 m); 2 ej., XII/93 (6 m); 2 ej., I/94 (5 m); 1 ej., II/94 (5 m). E 37: 4 ej., VIII/91 (6 m); 1 ej., IX/91 (10 m). E 38: 3 ej.,VII/91 (12 m); 1 ej., VIII/91 (16 m). E 40: 3 ej., VIII/91 (3-6 m). 42: 6 ej., III/91 (intermareal). E 43: 2 ej., VII/90 (33 m); 1 ej., V/93 (9 m); 4 ej., VII/93 (9 m); 1 ej., IX/93 (10 m); 1 ej., II/94 (8 m). E 44: 2 ej., VI/91 (8 m); 4 ej., VIII/91 (4 m); 1 ej., V/93 (4 m); 5 ej., VII/93 (8 m); 1 ej., X/93 (17 m). E 45: 1 ej., VII/93 (14 m).

CITAS BIBLIOGRÁFICAS: Bahía de Algeciras (Aartsen et al., 1984).

El tamaño de los ejemplares recolectados ha variado entre $3,3 \times 2,1 \mathrm{~mm}$ y $16 \times 10,2 \mathrm{~mm}$. El aspecto general del animal es ovalado, algo más estrecho en la parte caudal, con conchas consistentes. Se encuentran ornamentados con costillas longitudinales en el área central. El perinoto está constituido por escamas romboidales imbricadas, que ofrecen un aspecto de piel de serpiente.

Se encuentra en los mismos hábitat que Chiton olivaceus, aunque es menos abundante.

\section{Chiton phaesolinus (Monterosato, 1879)}

MATERIAL estudiado: E 30: 2 ej., VII/90 (6 m); 1 ej., II/91 (8 m); 1 ej., VII/91 (intermareal). E 32: 1 ej., V/91 (4-6 m); 2 ej., VIII/91 (intermareal). E 33: 1 ej., VIII/91 (12 m). E 34: 3 ej., VII/91 (4 m); 1 ej., IX/92 (8 m); 3 ej., VI/93 (8-10 m); 1 ej., VII/93 (10 m); 6 ej., VIII/93 (8 m); 1 ej., X/93 (12 m); 1 ej., II/94 (5 m). E 35: 2 ej., XI/91(5 m); 2 ej., I/93 (8 m); 1 ej., IX/93 (12 m); 2 ej., X/93 (5 m); 2 ej., XI/93 (5 m); 1 ej., XII/93 (5 m). E 37: 19 ej., VII/93 (8 m); 2 ej., X/93 (10 m). E 38: 1 ej., VIII/91 (16 m).

CitAs BIBLIOGRÁFICAS: Bahía de Algeciras (Aartsen et al., 1984).

Los ejemplares son de tamaño medio, cuyas dimensiones varían entre $3,1 \times 2,2 \mathrm{~mm}$ hasta 7,3 x $4,1 \mathrm{~mm}$. El aspecto general es ovalado, no carenado, bastante redondeado, con conchas no muy consistentes. Presenta un aspecto granulado fino, con 2-3 costillas no muy marcadas en las áreas pleurales. El perinoto está constituido por espículas romboidales imbricadas.

Se han hallado adheridos a piedras sueltas de fondos rocosos, generalmente provistas de algas calcáreas. Los ejemplares se encuentran de forma aislada, no asociados con otras especies de poliplacóforos.

\section{Suborden ACANTHOCHITONINA Bergenhayn, 1930 \\ Familia ACANTHOCHITONIDAE Pilsbry, 1893 Subfamilia ACANTHOCHITONIDAE Pilsbry, 1893}

\section{Acanthochitona fascicularis (Linneo, 1767)}

Material estudiado: E 01: 1 ej., VII/94 (8 m). E 02: 1 ej., VIII/93 (7 m). E 03: 2 ej., VII/93 (17 m). E 05: 2 ej., VIII/88 (intermareal). E 30: 1 ej., VI/91 (intermareal); 1 ej., VII/91 (15 m); 2 ej., IX/91 (3 m); 2 ej., IV/92 (3 m); 7 ej., IV/92 (8m); 1 ej.,. I/93 (15 m). E 31: 1 ej., VI/91 (3-12 m); 2 ej., VII/91 (12 m); 5 ej., VIII/91 (12 m); 17 ej., IX/92 (12 m). E 32: 3 ej., V/91 (intermareal); 1 ej., VIII/91 (6 m). E 33: 1 ej., 5/91 (12 m); 1 ej., VII/91 (12 m); 12 ej., IV/92 (9 m); 1 ej., VIII/92 (10 m). E 34: 1 ej., VII/91 (12 m); 13 ej., VII/91 (6 m); 2 ej., VII/91 (3 m); 1 ej., XI/91 (12 m); 24 ej., IX/92 (6 m). E 35: 1 ej., II/91(12 m); 20 ej., IX/92 (12 m); 86 ej., I/93 (5 m). E 37: 3 ej., VII/91 (10 m); 3 ej., VII/91 (3 m); 9 ej., VIII/91 (5 m); 1 ej., VII/92 (10 m). E 39: 1 ej., V/91 (16 m). E 40: 1 ej., VII/91 (3-6 m); 1 ej., VIII/92 (2 m). E 41: 1 ej., VIII/91 (18 m). E 42: 18 ej., II/91 (intermareal). E 43: 1 ej., VII/90 (2-33 m); 2 ej., VII/91 (30-33 m); 3 ej., VII/91 (4 m); 1 ej., IV/93 (9-11 m); 1 ej., V/93 (5-9 m); 1 ej., VI/93 (8 m); 1 ej., X/93 (10 m). E 44: 1 ej., VI/91 (17 m); 1 ej., VII/91 (4 m); 1 ej., VIII/91 (6 m). E 45: 4 ej., III/93 (20-25 m); 3 ej., IX/93 (9 m).

Citas bibliográficas: Portugal (Forbes y Hanley, 1853; Dell'Angelo y Gargiullo, 1978; Reis y Monteiro, 1984); Gibraltar (MacAndrew, 1850; Monterosato, 1888; Pilsbry, 1892); costas atlánticas de la Península Ibérica (Monterosato, 1879; Malatesta, 1962; Sabelli, 1974; Kaas, 1985); Algeciras (Carus, 1883; Aartsen et al., 1984); Algeciras y Gibraltar (Hidalgo, 1867; 1917); Ría de Faro, Olhao (Nobre, 1932; 1938).

El tamaño de los ejemplares ha oscilado entre 2,5 × 2 y $60 \times 25 \mathrm{~mm}$. La concha es oval alargada, carenada y con la pendiente de los lados rectas. Los ápices están fuertemente marcados y se imbrican sobre el seno jugal de la valva contigua. La ornamentación en la zona jugal es de apariencia lisa, aunque está constituida por cordones longitudinales. La zona pleurolateral presenta tubérculos redondeados convexos dispuestos sobre un pequeño pedúnculo y organizados en quincunce. El perinoto es ancho y se incrusta entre las suturas de las valvas, está ergido por espículas alargadas. En la zona sutural y cefálica se disponen, generalmente 18 penachos de largas espículas. El fleco marginal es generalmente patente. El articulamento no presenta líneas de inserción, sino únicamente escotaduras en número de 5/1/2. 
Se ha recolectado en fondos rocosos y de playas arenosas. Es muy abundante en la provincia de Cádiz, su presencia va disminuyendo desde el litoral de Huelva hasta Sagres.

\section{Acanthochitona crinita (Pennant, 1777)}

Material estudiado: E 01: 1 ej., ViI/94 (8 m). E 07: 2 ej., VI/94 (intermareal). E 27: 2 ej., IV/93 (intermareal). E 29: 1 ej., XII/91 (intermareal). E 30: 1 ej., V/91 (intermareal); 4 ej., VI/91 (intermareal). E 34: 1 ej., IX/91 (21 m); 2 ej., IX/92 (12 m); 1 ej., VII/93 (7-10 m). E 35: 2 ej., VI/93 (5 m); 1 ej., VII/93 (7-10 m). E 41: 1 ej., III/91 (2 m). E 42: 1 ej., VIII/91 (intermareal). E 43: 1 ej., VI/91 (30 m); 1 ej., IV/93 (9-11 m).

Citas Bibliográficas: Portugal (Bucquoy et al., 1882; Malatesta, 1962; Bosch y Sastre 1976; Kaas, 1985); Gibraltar (Jeffreys, 1882; Carus, 1883; Pilsbry, 1892; Maluquer, 1915); Algeciras y Gibraltar (Hidalgo, 1917); Faro, Culatra, Lagos (Nobre, 1932; 1938); Atlántico Español y Portugal (Yakovleva, 1952); Algarve (Reis y Monteiro, 1984); Algeciras (Aartsen et al., 1984).

El tamaño de los ejemplares ha variado entre 3 x 2 y 23 x $16 \mathrm{~mm}$. La forma de los ejemplares es oval alargada, siendo siempre el doble de largo que de ancho. Las valvas son carenadas con los bordes laterales lisos y con el ápice muy marcado, el cual se superpone sobre el seno jugal de la valva continua. La ornamentación del tegmento en la valva cefálica y zona pleurolateral está constituida por gránulos con forma de gota, aunque varía de gotas ligeramente redondeadas a alargadas y a su vez también varía la densidad de los gránulos, de manera que se pueden disponer muy juntos o bastante separados entre ellos. La zona jugal no muestra ornamentación, aunque se pueden apreciar en algunos ejemplares cordones longitudinales. El perinoto, que recubre parte de las valvas, puede presentar una apariencia desde aterciopelada a espinosa. En el primer caso el fleco marginal es patente, mientras que en el segundo apenas se aprecia; lo mismo sucede con los penachos suturales, denotándose fuertemente en los ejemplares de aspecto aterciopelado. En general presentan una gran variabilidad intraespecífica.

Se han encontrado principalmente, en piedras de playas arenosas y de roquedos. Se suele encontrar asociados con ejemplares de L. cinerea.

\section{Conclusiones}

En el suratlántico de la Península Ibérica, únicamente se habían citado 14 especies, con el presente trabajo se amplía hasta 20 por la presencia Callochiton septemvalvis, Lepidochitona montero- satoi, L. simrothi, L. canariensis, L. kaasi y L. severianoi, que no habían sido citadas en la zona de estudio.

Con las capturas de Callochiton septemvalvis y Lepidochitona monterosatoi en aguas suratlánticas ibéricas, se amplía el área de distribución en el atlántico ibérico, para ambas especies. Y el hallazgo de Lepidochitona simrothi y Lepidochitona canariensis, constituyen las primeras citas para la Península Ibérica.

\section{Referencias}

Aartsen, J., Menkhorst H. y Gittenber, E., 1984. Marine Mollusca from Algeciras. Basteria, 2: 1-135.

Bogi, G., Coppini, M. y Margelli, A., 1980. Contribution to the knowledge of the molluscan fauna of the central Tyrrhene Sea, Poliplacophora: part 1. La Conchiglia, 12(140-141): 14-18.

BorJa, A.,1983. Sistemática de los moluscos marinos de la costa vasca. Clave de moluscos de la zona intermareal. Publicación de la Sociedad Cultural "Insub". San Sebastián. Publicación n 2.

Bosch, M. y SASTRE, A., 1976. Sobre la presencia y distribución de "Polyplacophora" en el litoral de Mallorca. Boletín de la Sociedad de Historia Natural de Baleares, 12: 25-32.

Bucquoy, M. M. E., Dautzenberg, P. H. y Dollfus G., 1882. Les Mollusques marins du Roussillon. Fascicule $1^{\circ}$. J. B. Baillière \& Fils. Paris. 508 pp.

Burnay P. L., 1986. Moluscos Testáceos Marinhos da Berlanga (Portugal). MPAT. Secretaria de Estado do Ambiente e dos Recursos Naturais. Serviço Nacional de Parques, Reservas e Conservaçao da Natureza, Lisboa. 64 pp.

Capellini, J., 1859. Catalogue des Oscabriones de la Mediterranée. Journal de Conchyglia, 2 ser., 3: 320328.

Carmona Zalvide, Ma P. y Urgorri, V., 1999. Catálogo anotado de los moluscos poliplacóforos de Galicia (NO de España). Nova Acta Científica Compostelana (Bioloxia), 9: 2589-277.

CARus, J. V., 1883. Prodromus Fauna Mediterranea 2, Brachiostomata, Mullusca, Tunicata, Vertebrata: IIX. Stuttgart, 854 pp.

D’Angelo, G. y Gargiullo, S. 1978. Guida alle conchiglie Mediterranee. Ed. Fabri. Milano, 224 pp.

Estacio, F., Carballo, J. L., Carmona, P. y Zurita, F., 1993. Preliminary study about the aquatic fauna from the Piedras river marshes (Huelva, SW Spain). Arquivos do Museo Bocage, 2(18): 337-343.

Forbes, E. y HANLEY, S., 1853-55. Hystory of British mollusca and their shells. 4. Vol. John Van Voorst. London. 
Hidalgo, J. G., 1867. Catalogue des coquilles marines des costes de l'Espagne et des Iles Baleares. Journal of Conchology, 15: 416.

Hidalgo, J. G., 1917. Fauna malacológica de España, Portugal y las Baleares: Moluscos Testáceos Marinos. Trabajos del Museo Nacional de. Ciencias. Naturales, serie Zoologica, 30: 1-752.

JEFFREYS, J., 1882. On the Mollusca procured during the "Lightning" and "Porcupine" Expeditions, 1868-70. Proceedings of the Zoological Society of London, 656-687.

KAAS, P., 1954. Notes on Loricata. 2. On the ocurrence of Chaetopleura fulva (Wood, 1815) on the eastern coast of Latin America. Basteria, 18(1-2): 14-17.

KAAs, P., 1985. The genus Acanthochitona Gray, 1821 (Molusca, Polyplacophora) in the north-eastern Atlantic Ocean and in the Mediterranean Sea, with designation of neotypes of $A$. fascicularis (L., 1767) and of A. crinita (Pennant, 1777). Bulletin du Muséum d'Histoire Naturelle, Paris, 3: 579-609.

KaAs, P. y Van Belle, R. A., 1980. Catalog of living chitons (Mollusca: Polyplacophora). Dr. W. Publisher. Rotterdam. 144 pp.

KaAs, P. y Van Belle, R. A., 1981. The genus Lepidochitona Gray, 1821 (Mollusca: Polyplacophora) in the northeastern Atlantic Ocean, the mediterranean Sea and Blakc sea. Zoologische Verhandelingen, 185: $1-43$.

KaAs, P. y VAN Belle, R. A., 1985a. Monogroph of living chitons. 1, Order Neoloricata: Lepidopleurina. E. J. Brill/W. Backhuys. Leiden. 240 pp.

KaAs, P. y VAN Belle, R. A., 1985b. Monograph of living chitons. 2, Suborder Ischnochitonina, Ischnochitonidae: Schizoplacinae, Callochitoninae y Lepidochitoninae. E. J. Brill/W. Backhuys. Leiden. $198 \mathrm{pp}$.

KaAs, P. y VAn Belle, R. A., 1987. Monograph of living chitons. 3, Ischnochitonidae: Chaetopleurinae, Ischnochitoninae. E. J. Brill/ W. Backhuys. Leiden. $302 \mathrm{pp}$.

Laghi, G. F., 1977. Polyplacophora (Mollusca) neogenici dell'Appennino Settentrionale. Bolletino della Società Paleontologica Italiana, 16(1): 87-115.

LoCARD, A., 1886. Catalogue general des Mollusques vivants de France- Mollusques marines. H. George. Lyon. 778 pp.

MACANDREw, R., 1849. On the Mollusca of Vigo Bay in the North-West of Spain. Annals and Magazine of Natural History, 2(3): 507-512.

MACANDREW, R., 1850. Notes on the distribution and range in depth of Mollusca and other marine animals observed on the coast of Spain, Portugal, Barbary, Malta, and Southern Italy in 1849. Report of the 20th meeting of the British Association for the advancement of science: 264-304.
MalatestA, A., 1962. Mediterranean Polyplacophora Cenozoic and Recent. Geologia Romana, 1: 145171.

Maluquer, J., 1915. Amfineures de Catalunya. Treballs de l'Institucio Catalana d'Història Natural, 1: 187280.

Monterosato, T. A. DI, 1879. Enumerazione e sinonimia delle Conchiglie mediterranee - Monografia dei Chitonidi del Mediterraneo. Giornale di Scienze Naturali ed Economiche, 14: 9-31.

Monterosato, T. A. DI, 1888. Coquilles marines Marocaines. Journal de Conchyliologie, 20-40.

Nobre, A., 1932. Moluscos Marinhos de Portugal. Institute de Zoology do University de Porto. Porto. $466 \mathrm{pp}$.

Nobre, A., 1938-40. Fauna malacological del Portugal, Moluscos Marinhos e das aguas salobras. Companhia Editora do Minho. Porto. 894 pp.

PiAnI, P., 1980. Catalogo dei molluschi conchiferi viventi nel Mediterraneo. Bollettino malacologico, 16(56): 113-224.

Pilsbry, H. A., 1892-94. Monograph of the Polyplacophora. In: G.W. Tryon, Manual of Conchology, vol. 1 Academy of Natural Sciences. Philadelphia. $349 \mathrm{pp}$.

ReEve, L., 1847-48. Monograph of the genus Chiton. In: Conchologia Iconica. Shells of Molluscous Animals. London. 13.

Reis, C. A. y Montero, A. J., 1984. Aspectos ecologicos dos polyplacophora (Mollusca): da costa portuguesa. Actas do IV Simposio Iberico de Estudos do Benthos Marinhos, 1: 219-227.

SABelli, B. A., 1974. Origine e distribuzione dei Poliplacophora viventi in Mediterraneo. Quaderno civico della Stazione Idrobiologica di Milano, 5: 71-78.

Thiele, J., 1929. Handbuch der systematischen Weichtierkunde. Loricata. Rüdiger Bieler and Paula M. Mikkelsen. Washington. $34 \mathrm{pp}$.

Wood, W., 1815.Multivalve shells. Genus 1. Chiton. Generic character. General Conchology. 1-24.

YAKovleva, A. M., 1952. Shell-bearing mollusks (Loricata) of the seas of the U.S.S.R. - Fauna USSR. 45: 1-107. (Zool. Inst. Akad. Sci. USSR, Moskva and Leningrad). Translated into English by the Israel Program for Scientific Translations, Jerusalem. 
Apéndice.- Localidades de muestreo.

Appendix.- Collecting stations.

E 01.- Praia Mareta (Sagres, Portugal), $\left(37^{\circ} \mathrm{N} ; 08^{\circ} 56^{\prime} \mathrm{W}\right)$ Infralitoral rocoso con piedras a $8 \mathrm{~m}$ de profundidad.

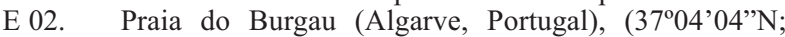
$08^{\circ} 49^{\prime} \mathrm{W}$ ): Infralitoral rocoso con piedras y arenales a 7 $\mathrm{m}$ de profundidad.

E 03. Praia do Lagos (Algarve, Portugal), $\left(37^{\circ} 10^{\prime} \mathrm{N}\right.$; $08^{\circ} 40^{\prime} \mathrm{W}$ ): Infralitoral rocoso en fondos de arena a $17 \mathrm{~m}$ de profundidad.

E 04. Praia da Rocha (Algarve, Portugal), (37 $07^{\prime} 11^{\prime \prime}$; $\left.08^{\circ} 35^{\prime} 14^{\prime \prime} \mathrm{W}\right)$ : Intermareal arenoso con promontorios rocosos

E 05. Praia Marinha (Algarve, Portugal), (37 $04^{\prime} 20^{\prime \prime}$; $08^{\circ} 24^{\prime} \mathrm{W}$ ): Intermareal arenoso con promontorios rocosos.

E 06. Almançao de Pera (Algarve, Portugal), $\left(37^{\circ} 10^{\prime} \mathrm{N}\right.$; $\left.08^{\circ} 20^{\prime} \mathrm{W}\right)$ : Arenal infralitoral con salientes rocosos, 22 $m$ de profundidad.

E.07. Praia do Hollos (Algarve, Portugal), $\left(37^{\circ} 05^{\prime} \mathrm{N}\right.$; $\left.08^{\circ} 05^{\prime} \mathrm{W}\right)$ : Intermareal rocoso con charcas litorales y algas.

E 08. Vilareal Sto Antonio (Algarve, Portugal), $\left(37^{\circ} 10^{\prime} \mathrm{N}\right.$; $\left.07^{\circ} 25^{\prime} \mathrm{W}\right)$ : Muestra obtenida en las redes de los pescadores.

E 09. Isla Cristina (Huelva), $\left(37^{\circ} 11^{\prime} 53^{\prime} \mathrm{N} ; 07^{\circ} 11^{\prime} \mathrm{W}\right)$ Intermareal arenoso con cascajos.

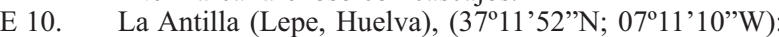
Fondo de cascajos y arena a $14 \mathrm{~m}$ de profundidad.

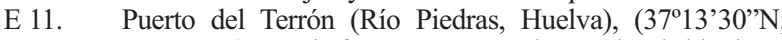
$\left.07^{\circ} 10^{\prime} 24^{\prime \prime}\right)$ : Fondo fangoso con conchas vacias de bivalvos

E 12. Isla del Vinagre (Río Piedras, Huelva), (3709'48”N $\left.07^{\circ} 12^{\prime} 42^{\prime \prime} \mathrm{W}\right)$ : Fondo areno-fangoso con conchas de moluscos.

E 13. Casa Capilar (Río Piedras, Huelva), $\left(37^{\circ} 12^{\prime} 48^{\prime \prime N}\right.$; $\left.07^{\circ} 08^{\prime} 42^{\prime \prime} \mathrm{W}\right)$ : Fondo fangoso a $3.5 \mathrm{~m}$ de profundidad.

E 14. Caño Tendal (Río Piedras, Huelva), $\left(37^{\circ} 13^{\prime} 06^{\prime \prime} \mathrm{N}\right.$; $\left.07^{\circ} 07^{\prime} 54^{\prime \prime} \mathrm{W}\right)$ : Fondo fangoso.

E 15. Embarcadero del Rompido (Río Piedras, Huelva), $\left(37^{\circ} 13^{\prime} 06 \mathrm{~N} ; 07^{\circ} 07^{\prime} 12^{\prime \prime} \mathrm{W}\right)$ : Fondo fangoso con conchas de bivalvos.

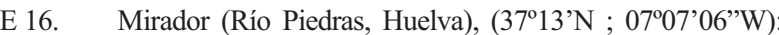
Fondo areno-fangoso a $5 \mathrm{~m}$ de profundidad.

E 17. La Galera (Río Piedras, Huelva), $37^{\circ} 13^{\prime} 12^{\prime \prime} \mathrm{N} ; 07^{\circ} 06^{\prime} \mathrm{W}$ ) Fondo areno-fangoso a $5 \mathrm{~m}$ de profundidad.

E 18. Aguas del Pino (Río Piedras, Huelva), $\left(37^{\circ} 12\right.$ '54”N $\left.07^{\circ} 05^{\prime} 06^{\prime \prime} \mathrm{W}\right)$ : Intermareal areno - fangosa con piedras aisladas.

E 19. Caño la Culata (Río Piedras, Huelva), $\left(37^{\circ} 12^{\prime} 50^{\prime}{ }^{\prime}\right.$; $\left.07^{\circ} 02^{\prime} 51^{\prime \prime} \mathrm{W}\right)$ : Intermareal arenoso - fangoso con cantos rodados y acúmulos de conchas.

E 20. El Portil (Desembocadura del Río Piedras, Huelva), $\left(37^{\circ} 12^{\prime} 40^{\prime \prime} \mathrm{N} 07^{\circ} 02^{\prime} 50^{\prime \prime} \mathrm{W}\right)$ : Intermareal con promontorios rocosos y numerosas anfractuosidades entre los arenales costeros.

E 21. Mazagón (Huelva), (36 $\left.56^{\circ} \mathrm{N} ; 06^{\circ} 50^{\prime} 3^{\prime} \mathrm{W}\right)$ : Fondo de cascajo arenoso a $35 \mathrm{~m}$ de profundidad.

E 22. Puerto Real (Desembocadura del Río San Pedro, Cádiz), $\left(36^{\circ} 31^{\prime} 50^{\prime \prime} \mathrm{N} ; 06^{\circ} 13^{\prime} 20^{\prime \prime} \mathrm{W}\right)$ : Intermareal areno-fangoso con piedras sueltas

E 23. Bahía de Cádiz, $\left(36^{\circ} 30^{`} \mathrm{~N} ; 06^{\circ} 15^{\prime} \mathrm{W}\right)$ : Intermareal areno-fangoso, con promontorios de rocas y piedras sueltas.

E 24. El Arrecifillo (Conil, Cádiz), (36 $\left.16^{\circ} 80^{\prime \prime} \mathrm{N} ; 06^{\circ} 08^{\prime} 05^{\prime \prime} \mathrm{W}\right)$ Infralitoral rocoso con grandes piedras a $12 \mathrm{~m}$ de profundidad.

E 25. Cabo de Trafalgar (Cádiz), $\left(36^{\circ} 08^{\prime} \mathrm{N}\right.$; $\left.05^{\circ} 48^{\prime} \mathrm{W}\right)$ : Intermareal arenoso con roquedos y promontorios pedregosos
E 26. Arrecife artificial (Conil, Cádiz), (36²10’30" N; $\left.06^{\circ} 15^{\prime} 60^{\prime \prime} \mathrm{W}\right)$ : Estructuras de hormigón artificiales, sobre fondo de arena y cascajos a $28 \mathrm{~m}$ de profundidad.

E 27. Caños de Meca (Cádiz), (36 $10^{\circ} 40^{\prime \prime} \mathrm{N} ; 6^{\circ} 10^{\prime} \mathrm{W}$ ): Intermareal arenoso con zonas de roca y con piedras sueltas y charcas de marea.

E 28. Isla del Tajo (Barbate, Cádiz), $\left(36^{\circ} 10^{\prime} 60^{\prime \prime} \mathrm{N}\right.$; $\left.05^{\circ} 58^{\prime} 75^{\prime \prime} \mathrm{W}\right)$ : Grandes promontorios rocosos infralitorales con fuerte cobertura algal a $8 \mathrm{~m}$ de profundidad.

E 29. Playa del Chorro (Barbate, Cádiz), $\left(36^{\circ} 10^{\prime} 55^{\prime \prime} \mathrm{N}\right.$; $\left.05^{\circ} 58^{\prime} 50^{\prime \prime} \mathrm{W}\right)$ : Intermareal rocoso, con piedras y charcas de mareas.

E 30. Isla de Tarifa (Tarifa, Cádiz), (3601' ' $" N$; 0536'22”W): Roquedo con charcas intermareales, presentando gran cobertura algal de Enteromorpha sp. y algas calcáreas; infralitoral rocoso desde $3 \mathrm{~m}$ hasta $20 \mathrm{~m}$ de profundidad.

E 31. Isla de Las Palomas (Algeciras, Cádiz), (3604'N; $05^{\circ} 26^{\prime} \mathrm{W}$ ): Infralitoral rocoso desde $3 \mathrm{~m}$ hasta $12 \mathrm{~m}$ de profundidad.

E 32. Punta Carnero (Algeciras, Cádiz), (3604'65”N; $\left.05^{\circ} 25^{\prime} 40^{\prime \prime} \mathrm{W}\right)$ : Intermareal e infralitoral rocoso con piedras, desde $1 \mathrm{~m}$ hasta $18 \mathrm{~m}$ de profundidad.

E 33. La Ballenera (Ensenada de Getares, Algeciras, Cádiz), $\left(36^{\circ} 05^{\prime} 40^{\prime \prime} \mathrm{N}\right.$; $\left.05^{\circ} 26^{\prime} 09^{\prime \prime} \mathrm{W}\right)$ : Infralitoral rocoso con piedras y arenales desde $2 \mathrm{~m}$ hasta $12 \mathrm{~m}$ de profundidad

E 34. Punta de San García (Algeciras, Cádiz), $\left(36^{\circ} 06^{\prime} 04^{\prime} N\right.$; $05^{\circ} 25^{\prime} 1$ ' W): Infralitoral con grandes y abundantes promontorios rocosos y con zonas de arena y piedras, entre 2 y $12 \mathrm{~m}$ de profundidad.

E 35. Ensenada de Cucareo (Algeciras, Cádiz), $\left(36^{\circ} 07^{\prime} \mathrm{N}\right.$; $05^{\circ}$ $\left.25^{\prime} \mathrm{W}\right)$ : Infralitoral arenoso con abundantes piedras sueltas, variando la profundidad entre 2 y $12 \mathrm{~m}$.

E 36. Dársena del Saladillo (Algeciras, Cádiz), (3707'20”N; $\left.05^{\circ} 25^{\prime} 37^{\prime \prime} \mathrm{W}\right)$ : Fondos biodetríticos fangosos de 1,5 a 5 $m$ de profundidad.

E 37. Puerto de Algeciras (Algeciras, Cádiz), (3607’35”N; $05^{\circ} 25^{\prime} 95^{\prime}$ 'W): Fondos biodetríticos, la profundidad ha oscilado entre 3 y $10 \mathrm{~m}$.

E 38. Punta de Paredones (Algeciras, Cádiz), ( $36^{\circ} 08^{\prime} \mathrm{N}$; $\left.05^{\circ} 26^{\prime} 50^{\prime \prime} \mathrm{W}\right)$ : Infralitoral rocoso, con zonas de arenas, desde 3 hasta $16 \mathrm{~m}$ de profundidad.

E 39. El Rinconcillo (Algeciras, Cádiz), (3609'11'N; $05^{\circ} 25^{\prime} 50^{\prime \prime} \mathrm{W}$ ): Fondos de arena, con grandes promontorios rocosos, en los que abundan piedras sueltas, la profundidad ha variado entre 2 y $25 \mathrm{~m}$.

E 40. Punta del Gallo del Mirador (Algeciras, Cádiz), $\left(36^{\circ} 10^{\prime} \mathrm{N}\right.$; $\left.05^{\circ} 24^{\prime} 50^{\prime \prime} \mathrm{W}\right)$ : Infralitoral de roquedos, asociados a fondos de arena, entre 3 y $22 \mathrm{~m}$ de profundidad.

E 41. Refinería CEPSA (Algeciras, Cádiz), (36¹1'70”N; $\left.05^{\circ} 23^{\prime} 80^{\prime \prime} \mathrm{W}\right)$ : Infralitoral con fondos biodetríticos, entre 2 y $20 \mathrm{~m}$ de profundidad.

E 42. Campamento (Algeciras, Cádiz), (36²10'50”N; $05^{\circ} 23^{\prime} 30^{\prime \prime} \mathrm{W}$ ): Roquedo intermareal, con grandes piedras $\mathrm{y}$ charcas de mareas.

E 43. Crinavis (Algeciras, Cádiz), $\left(36^{\circ} 10^{\prime} \mathrm{N} ; 05^{\circ} 23^{\prime} \mathrm{W}\right)$ : Roquedo infralitoral, rodeado de arenales, variando la profundidad desde 2 hasta $33 \mathrm{~m}$.

E 44. San Felipe (La Línea, Cádiz), (36²10'80”N; $\left.05^{\circ} 21^{\prime} 73^{\prime \prime} \mathrm{W}\right)$ : Infralitoral de fondos biodetríticos, entre 2,5 y $17 \mathrm{~m}$ de profundidad.

E 45. Gibraltar, $\left(36^{\circ} 08^{\prime} \mathrm{N} ; 05^{\circ} 21^{\prime} \mathrm{W}\right)$ : Infralitoral rocoso, entre 20 y $25 \mathrm{~m}$ de profundidad. 